\title{
Escala do controle da ação para o diagnóstico de desempenho em estudantes de Enfermagem
}

\author{
The action control scale for diagnosing performance in nursing students
}

Escala de control de la acción para diagnóstico de rendimiento en estudiantes de Enfermería

\author{
Elen Martins da Silva Castelo Branco', Mauricio Abreu Pinto Peixoto", Neide Aparecida Titonelli Alvim' \\ ' Universidade Federal do Rio de Janeiro, Escola de Enfermagem Anna Nery, \\ Departamento de Enfermagem Fundamental. Rio de Janeiro-RJ, Brasil. \\ "Universidade Federal do Rio de Janeiro, Núcleo de Tecnologia Educacional para a Saúde. Rio de Janeiro-RJ, Brasil.
}

\section{Submissão: 05-08-2012 Aprovação: 18-11-2013}

\section{RESUMO}

Pesquisa quantitativa que objetivou verificar a pertinência da Teoria do Controle da Ação para a identificação do risco para desempenho insatisfatório no planejamento dos cuidados preventivos para úlceras por pressão. A orientação ação-estado trata das diferenças individuais e da capacidade de regular as emoções, cognições e atitudes para concluir as ações intencionais. Para a coleta dos dados, o Instrumento de Avaliação Discente e o Questionário HAKEMP 90, derivado da referida teoria, foram aplicados em 46 estudantes da Graduação em Enfermagem. A análise evidenciou sensibilidade elevada $(0,84)$ para o diagnóstico do risco para desempenho insatisfatório e especificidade elevada $(0,90)$ para a detecção da ausência do risco. Os resultados recomendam o questionário HAKEMP 90 como recurso diagnóstico, por identificar elementos essenciais para a formação do enfermeiro, como a aprendizagem ativa, a regulação dos processos cognitivos e a relevância dos cuidados fundamentais de enfermagem no ambiente hospitalar.

Descritores: Enfermagem; Aprendizagem; Úlcera por Pressão.

\begin{abstract}
Quantitative research aimed to verify the relevance of Action Control Theory for the identification of risk for poor performance in the planning of preventive care for pressure ulcers. The action - state orientation deals with individual differences and the ability to regulate emotions, cognitions and attitudes to complete the intentional actions. The instruments used were the Student Assessment Instrument and HAKEMP 90, derived from this theory, in 46 undergraduate nursing students. The analysis showed high sensitivity (0.84) for the diagnosis of risk for poor performance and high specificity (0.90) for detecting the absence of risk in the care planning. The results suggest the HAKEMP 90 as a diagnostic tool for identifying essential elements of nursing education as active learning, regulation of cognitive processes and relevance of basic nursing care in hospital.
\end{abstract}

Key words: Nursing; Learning; Pressure Ulcer.

\section{RESUMEN}

Investigación cuantitativa desarrollada con fin de verificar la pertinencia de la Teoría del Control de Acción para identificación de riesgos de bajo desempeño en planificación de atención preventiva de úlceras por presión. La orientación acción - estado describe diferencias individuales y capacidad de regular emociones, cogniciones y actitudes para completar acciones intencionales. El Instrumento de Evaluación de Estudiantes y el Cuestionario HAKEMP 90 se aplicaron en 46 estudiantes de enfermería. El análisis demostró alta sensibilidad $(0,84)$ para el diagnóstico de riesgo de bajo rendimiento y alta especificidad $(0,90)$ para detectar la ausencia de riesgo. Los resultados sugieren que la HAKEMP 90 como herramienta de diagnóstico para la identificación de los elementos esenciales de la educación de enfermería como el aprendizaje activo, la regulación de los procesos cognitivos y la relevancia de los cuidados básicos de enfermería en el hospital.

Palabras clave: Enfermería; Aprendizaje; Ulcera por Presión. 


\section{INTRODUÇÃO}

O artigo trata da aplicação da Escala do Controle da Ação (HAKEMP 90) em estudantes de enfermagem com o propósito de verificar a pertinência da Teoria do Controle da Ação (TCA) (1-2) para a identificação precoce do risco para desempenho insatisfatório no planejamento dos cuidados de enfermagem preventivos para úlceras por pressão (UP).

A úlcera por pressão é definida como uma lesão na pele e/ ou tecido subjacente, geralmente localizada em proeminências ósseas e que tem como causas a pressão, o cisalhamento ou a fricção ${ }^{(3)}$. Apesar de ser uma lesão evitável, apresenta alta incidência tanto em pacientes que recebem cuidados no domicilio como aqueles internados em instituições hospitalares ${ }^{(4)}$ e configura-se uma questão desafiadora para a equipe multiprofissional de saúde.

O sofrimento decorrente deste agravo para os clientes hospitalizados e seus familiares é imenso no que se refere ao aumento da permanência hospital, às limitações nas atividades diárias e no bem-estar em geral. Ainda mais, as múltiplas modalidades terapêuticas indicadas são impactantes para os custos hospitalares ${ }^{(5-6)}$. Assim sendo, a etiologia da úlcera por pressão e as medidas preventivas devem ser discutidas enfaticamente na formação do enfermeiro, reafirmando-se a sua importância ao longo do exercício profissional.

A sistematização dos cuidados de enfermagem para a prevenção das UP requer dos estudantes habilidades e competências essenciais para a avaliação dos fatores de risco, das alterações fisiopatológicas e psicossociais que emergem da clientela. A rapidez e o silêncio com que se instalam as lesões demandam um exame minucioso e atento do cliente para a prevenção das complicações. Tanto o diagnóstico quanto o prognóstico das alterações da pele pressupõem o uso da atenção seletiva e do pensamento crítico na avaliação continua da clientela.

Entretanto, observa-se que há um distanciamento da atenção do estudante de enfermagem durante a avaliação da pele, principalmente quando se trata da pele íntegra em situação de risco. Consequentemente, sobrevêm as lacunas no diagnóstico e a prescrição fragmentada para a prevenção das lesões. A situação em si, revela aspectos interferentes e a existência destes, em maior ou menor grau, afeta a concentração e impede ou dificulta o curso das ações durante a atividade em tela. Em suma, a descrição clara e objetiva dos achados no exame físico, o diagnóstico de enfermagem, o planejamento e a avaliação continua do cliente são ações que demandam a sensibilidade, a concentração e a atenção, e também o uso coerente dos princípios fundamentais e dos instrumentos básicos de enfermagem.

Assim, é imprescindível que o estudante de enfermagem aprenda a planejar os cuidados preventivos para UP o mais conscientemente possível. Essa consciência permite ao mesmo tempo, a avaliação das necessidades individuais, a prescrição dos cuidados de acordo com as evidências constatadas e a reflexão sobre as próprias ações para aprimorá-las.

Para lidar com tais questões torna-se necessário agregar novas possibilidades ao processo educativo. A aproximação entre a TCA e a enfermagem é uma iniciativa inovadora que propicia uma reflexão critica sobre o desempenho do estudante visando à ampliação de recursos para a promoção de um olhar mais crítico sobre a aprendizagem individual e significativa e consequentemente, a sistematização da assistência mais efetiva.

\section{TEORIA DO CONTROLE DA AÇÃO}

A TCA explica como os estilos pessoais de controle da ação - orientação para a ação e estado interferem na regulação das ações humanas. É um constructo que trata das diferenças individuais e da capacidade para regular as emoções, cognições, comportamentos e estratégias autoreguladoras a fim de concluir as ações planejadas. O componente-chave da teoria é a intenção, ou seja, um propósito que orienta o sujeito para o alcance das suas metas. Ressalta-se ainda na TCA, o papel mediador do controle volitivo entre a intenção inicial e a ação concreta, perpassando por elementos que situam o modo de agir do sujeito no momento presente e no planejamento para as ações futuras ${ }^{(7)}$.

A orientação para a ação caracteriza-se pela tomada de decisão consciente, pelo uso de estratégias autoreguladoras coerentes com a atividade em si e pelo perfil emocional adaptado às situações de insucesso. A presença destes elementos permite a retomada mais efetiva do curso da ação. Nesse caso, as competências acadêmicas situam-se em um nível mais elevado com o predomínio do interesse intrínseco pelo objeto da aprendizagem. O estudante com uma forte orientação para a ação é um sujeito ativo no processo educativo e a criticidade na auto-avaliação possibilita mais claramente o planejamento das ações futuras orientadas para fins específicos. Neste tipo de orientação, o professor é o mediador da aprendizagem. Em síntese, os indivíduos orientados para a ação iniciam as ações de forma eficaz para alcançar as metas e permanecem mais concentrados até a conclusão da atividade ${ }^{(8)}$.

Por outro lado, a orientação para o estado demonstra a tendência para o declínio da persistência diante do insucesso acadêmico. O estudante mantém - se estático, as distrações desviam facilmente a atenção da atividade e a retomada do processo decisório é mais lenta. Outra característica marcante é a superficialidade na busca por informações, ou seja, na maioria dos casos ocorre a coleta dos dados mais simples e aparentes. O uso das estratégias autoreguladoras é predominantemente desorganizado.

Nesse sentido, a importância da atenção seletiva, a concentração, o conhecimento sobre o próprio modo de agir para alcançar os objetivos e o uso do conhecimento prévio são componentes que justificam o estudo da ação, pela capacidade de facilitar ou não a sua consecução ${ }^{(9)}$.

A lógica da TCA permite afirmar que o estudante ativo, concentrado e atento reúne, entre outras de igual valor, características essenciais para aprender e aqui, foram aplicados tais princípios para o planejamento dos cuidados preventivos para UP realizados por estudantes de enfermagem no ambiente hospitalar.

Considerando a contextualização apresentada emergiu a seguinte questão norteadora: a aplicação da Teoria do Controle da Ação permite identificar os estudantes com alto e baixo risco para o desempenho insatisfatório no planejamento dos cuidados de enfermagem para a prevenção das UP? Para 
orientar o estudo, foi estabelecido o objetivo de verificar a pertinência da Teoria do Controle da Ação na identificação precoce de estudantes de enfermagem com alto-risco para desempenho insatisfatório no planejamento dos cuidados fundamentais para a prevenção das UP.

\section{METODOLOGIA}

Trata-se de um estudo quantitativo desenvolvido no âmbito de um Hospital Universitário Federal no Rio de Janeiro. Participaram do estudo 46 estudantes, sendo 45 do sexo feminino e 01 do sexo masculino, cuja faixa etária variou entre 18 e 25 anos. Como critérios de inclusão, os estudantes deveriam estar matriculados no quinto período da Graduação em Enfermagem e desenvolvendo atividades curriculares no cenário hospitalar. Não houve exclusão de nenhum participante.

O estudo foi aprovado pelo Comitê de Ética em Pesquisa sob o Protocolo no 097/06 atendendo a Resolução 196/96. A coleta de dados se iniciou após leitura e assinatura do Termo de Consentimento Livre e Esclarecido. A participação voluntária e o anonimato foram preservados em todas as etapas do estudo.

Para a coleta de dados foram utilizados o Instrumento de Avaliação Discente (IAD), elaborado pelos autores do estudo, e o Questionário HAKEMP 90, derivado da TCA, e traduzido para a língua portuguesa. O IAD foi estruturado na forma de Escala Likert, com 18 enunciados que descreviam atitudes desejadas e características essenciais para a avaliação do estudante na tarefa especifica, durante o período letivo. A Escala do Controle da Ação foi aplicada após o término das atividades acadêmicas e da divulgação das notas obtidas pelos estudantes, de modo a assegurar-lhes que o preenchimento da Escala não iria interferir na avaliação final. Para a análise estatística foram utilizados os critérios de sensibilidade e especificadade para testes diagnósticos.

\section{RESULTADOS}

\section{Instrumento de avaliação discente}

O objetivo desta etapa foi classificar os estudantes em dois grupos, alto e baixo risco para desempenho insatisfatório. O IAD reuniu um conjunto de dezoito itens que descreviam habilidades e competências, as ações mais simples e mais gerais para o cuidado de enfermagem, os instrumentos básicos para cuidar e a as etapas da sistematização visando à prevenção das UP. Os enunciados foram avaliados por seis especialistas a fim de identificar, discutir e avaliar as dificuldades de compreensão conceitual e a adequação às características e atitudes essenciais ao estudante de enfermagem ao prevenir UP, citadas anteriormente.

Ressalta-se que o instrumento oferecia quatro opções em que se poderia assinalar a avaliação do desempenho do estudante na situação em estudo, considerando-o muito insatisfatório(MI), insatisfatório(I), satisfatório(S) ou plenamente satisfatório(PS). Os índices de desempenho individual (IAD) foram calculados ao efetuar-se a multiplicação do número de ocorrências registradas em cada enunciado pelo valor normativo do instrumento $(M I=1, I=2, S=3$ e $P S=4)$.

A título de exemplo, o índice de desempenho do estudante
EE33(O, 62) foi calculado efetuando-se a divisão da pontuação total obtida (45) pela máxima possível (72). Após o cálculo dos índices de desempenho, a mediana foi a medida aplicada para o corte dos escores individuais. Considerou-se como grupo de baixo risco ou de desempenho satisfatório (G1) os estudantes com mediana igual ou superior a 0,70 e como grupo alto risco ou desempenho insatisfatório (G2) a mediana inferior a este valor (Quadro1).

\section{Questionário HAKEMP 90}

O Questionário HAKEMP 90 apresenta 36 itens distribuídos em três subsescalas, com duas alternativas de resposta, uma indicando a orientação para a ação (OA) e outra a orientação para o estado (OE), e encontra-se disponível nas versões em inglês, espanhol e alemão(10-12). As subescalas investigam três dimensões em particular que se relacionam com o alcance das metas: a orientação para ação/estado após a situação de fracasso (OAF), orientação ação/estado no planejamento e decisão para a ação (OAP) e orientação ação/estado na atividade bem-sucedida (foco na atividade e na motivação intrínseca- OAA). Para o estudo optou-se pela tradução, retradução e adaptação transcultural da versão em espanhol e a equivalência para o português ${ }^{(13-14)}$.

A qualificação do conjunto de respostas de cada estudante foi obtida com a soma dos pontos, contida no intervalo entre zero e $12^{(12)}$. Na subescala OAF considera-se que a pontuação contida entre zero e 4 indica orientação para a ação e entre 5 e 12 para o estado. Na subescala OAP a pontuação entre zero e 5 corresponde à orientação para o estado(OE) e 6 e 12 para a ação. Nas normas para a escala OAA o valor contido entre 0 e 9 indica orientação para o estado e entre 10 e 12 para a ação.

Os índices calculados no IAD e a classificação do estudante para ação (OA) ou estado (OE) em cada subescala foram comparados e analisados segundo os critérios de sensibilidade e especificidade. O Quadro 1 apresenta os resultados obtidos a partir da aplicação dos instrumentos, identifica o grupo de risco composto por 22 estudantes e os respectivos índices compreendidos entre 0,53 e 0,69.

Quadro 1 - Comparação entre IAD e HAKEMP 90 para o desempenho insatisfatório (G2)

\begin{tabular}{|c|c|c|c|c|}
\hline \multirow{2}{*}{$\begin{array}{c}\text { ESTUDANTES COM } \\
\text { DESEMPENHO IN- } \\
\text { SATISFATÓRIO } \\
\text { (G2) }\end{array}$} & \multicolumn{3}{|c|}{ HAKEMP 90 } & \multirow{2}{*}{ IAD } \\
\cline { 2 - 4 } & OAF & OAP & OAA & \\
\hline EE37 & OA & OE & OA & 0,69 \\
\hline EE26 & OA & OE & OE & 0,68 \\
\hline EE07 & OE & OA & OE & 0,67 \\
\hline EE08 & OA & OA & OE & 0,67 \\
\hline EE15 & OE & OA & OE & 0,67 \\
\hline EE32 & OE & OE & OA & 0,67 \\
\hline EE46 & OA & OE & OE & 0,67 \\
\hline EE34 & OE & OE & OE & 0,65 \\
\hline EE18 & OE & OE & OA & 0,64 \\
\hline
\end{tabular}




\begin{tabular}{|c|c|c|c|c|}
\hline EE21 & OE & OE & OE & 0,63 \\
\hline EE44 & OE & OE & OE & 0,63 \\
\hline EE33 & OE & OA & OE & 0,62 \\
\hline EE12 & OA & OE & OE & 0,61 \\
\hline EE16 & OE & OE & OA & 0,61 \\
\hline EE23 & OE & OA & OE & 0,61 \\
\hline EE42 & OE & OE & OE & 0,61 \\
\hline EE10 & OE & OE & OE & 0,59 \\
\hline EE22 & OE & OA & OE & 0,59 \\
\hline EE31 & OE & OA & OA & 0,59 \\
\hline EE03 & OE & OE & OE & 0,56 \\
\hline EE05 & OA & OA & OE & 0,54 \\
\hline EE28 & OA & OE & OA & 0,53 \\
\hline
\end{tabular}

$O A$ - orientação para a ação. OE - orientação para o estado EE - estudante de enfermagem

\section{Sensibilidade(S) e especificidade (E)}

Visando à comprovação do objeto do estudo, utilizou-se o calculo da sensibilidade e da especificidade para comparar os grupos de baixo risco (G1) e alto risco (G2) com a orientação para a ação (OA) e estado (OE). A sensibilidade é a proporção de resultados verdadeiros positivos e expressa a probabilidade de um teste ser positivo, ou seja, é a medida da capacidade do método de decisão de predizer a condição que se deseja detectar. Um teste sensível é escolhido quando não se pode correr o risco de elaborar diagnósticos equivocados, a exemplo de uma condição perigosa ou uma doença grave. Ressalta-se que quanto mais sensível for o teste maior será o valor preditivo negativo, ou seja, a quase garantia de que a condição investigada está ausente.

A especificidade expressa a proporção de verdadeiros negativos, ou seja, a capacidade do método para indicar a ausência de uma certa condição. Os testes específicos são recomendados para confirmar um diagnóstico e raramente indicarão o resultado positivo na ausência da doença. Portanto, o teste diagnóstico que demonstra especificidade elevada indica poucos resultados falso-positivos. Quanto mais específico for um teste, melhor será o seu valor preditivo positivo ${ }^{(15-16)}$.

Para a análise inicial, os escores das três subescalas foram calculados separadamente, devido à correlação que guardam com os comportamentos distintos pela TCA. Os valores a partir de 0,80 determinaram a validade interna do teste, considerando que o valor obtido com a sensibilidade respondeu a questão prática do número de casos positivos ${ }^{(17)}$.

A subescala OAF permite identificar a orientação para ação ou estado dos estudantes diante do fracasso acadêmico e a execução eficiente ou não de uma nova tarefa após a experiência. A Tabela 1 se refere aos resultados da análise desta subescala. Evidencia-se a sensibilidade de 0,69 para a proporção de resultados verdadeiros positivos, a discreta diferença entre os valores obtidos para sensibilidade e especificidade $(0,056)$ e a variação semelhante tanto para o valor preditivo positivo(VPRP) quanto para o negativo(VPRN).
Tabela 1 - Sensibilidade e especificidade das subescalas OAF

\begin{tabular}{ccccc}
\hline \multicolumn{5}{c}{ Desempenho (md) } \\
\hline OAF & & $(\mathbf{G} 2)$ & $(\mathbf{G} 1)$ & Total \\
& OE & 12 & 9 & \\
& OA & 10 & 15 & 46 \\
& Total & 22 & 24 & 0,625 \\
& S $=$ & 0,691818 & VPRP $=$ & 0,681818 \\
\hline
\end{tabular}

A subescala OAP possibilita investigar a orientação para a ação ou estado diante do planejamento, da decisão para a ação e da coerência entre a intenção e a realização de fato. A Tabela 2 demonstra a capacidade de medida para a predição da condição de risco a partir dos índices de $\mathrm{S}=0,70$ e VPP $=$ 0,68 . Esses resultados indicaram que OAP mostrou-se sensível para o diagnóstico dos estudantes em risco para o desempenho insatisfatório relacionado ao planejamento das ações de enfermagem preventivas para UP.

Tabela 2 - Sensibilidade e especificidade das subescalas OAP

\begin{tabular}{ccccc}
\hline \multicolumn{5}{c}{ Desempenho } \\
\hline OAP & \multicolumn{3}{c}{ Total } \\
& OA & 17 & $(\mathbf{G} 2)$ & \\
& OE & 7 & 12 & 46 \\
& Total & 24 & 22 & 0,68 \\
& S= & 0,708333 & VPRP $=$ & 0,666667 \\
\hline
\end{tabular}

A subescala OAA pode ser concebida como um indicador do envolvimento e da motivação intrínseca do estudante na atividade, considerando como foco o alcance da meta. $\mathrm{Na}$ Tabela 3 destaca-se o valor da especificidade $(E=0,72)$ o que evidencia o baixo risco nos estudantes orientados para ação. A especificidade expressa a capacidade de o teste apontar a ausência da condição em estudo.

Tabela 3 - Sensibilidade e especificidade das subescalas OAA

\begin{tabular}{ccccc}
\hline \multicolumn{5}{c}{ Desempenho } \\
\hline OAA & & (G1) & (G2) & Total \\
& OA & 9 & 6 & \\
& OE & 15 & 16 & 46 \\
& Total & 24 & 22 & \\
& & & & 0,6 \\
& S $=$ & 0,375 & VPRP $=$ & 0,516129 \\
\hline E $=$ & 0,727273 & VPRN $=$ &
\end{tabular}


A partir dos resultados encontrados procedeu-se à leitura combinada das subescalas com o objetivo de selecionar o par mais sensível para os indicadores comportamentais investigados. A etapa deu visibilidade aos valores mais relevantes segundo a validade interna e as recomendações do autor ${ }^{(18)}$.

$\mathrm{Na}$ Tabela 4, a combinação entre as subescalas OAF e OAP mostrou-se sensível $(S=0,81 ; \mathrm{VPN}=0,86)$ para a detecção do risco de desempenho relacionado à ação em situações de insucesso, no planejamento e na tomada de decisão. Analisadas em conjunto, as subescalas OAP e OAA apontaram elevada especificidade $(E=0,90)$ para detectar a ausência de risco para o planejamento e a motivação intrínseca na atividade. As subescalas OAA e OAF apresentaram sensibilidade elevada $(S=0,84)$ para o diagnóstico dos estudantes em risco para desempenho insatisfatório relacionado ao envolvimento motivacional intrínseco e ao insucesso acadêmico no presente e as repercussões sobre eventos situações futuras.

Na combinação das escalas OAF e OAP o grupo de baixo risco (G1) totalizou dezoito estudantes entre os quais treze foram classificados segundo a orientação para a ação $(72,2 \%)$ e cinco para o estado $(27,8 \%)$. A análise também demonstrou que onze estudantes compõem o grupo de risco e destes, nove atendem as características da orientação para o estado $(81,8 \%)$ e apenas dois são orientados para a ação $(18,2 \%)$.

Tabela 4 - Sensibilidade e especificidade das subescalas combinadas

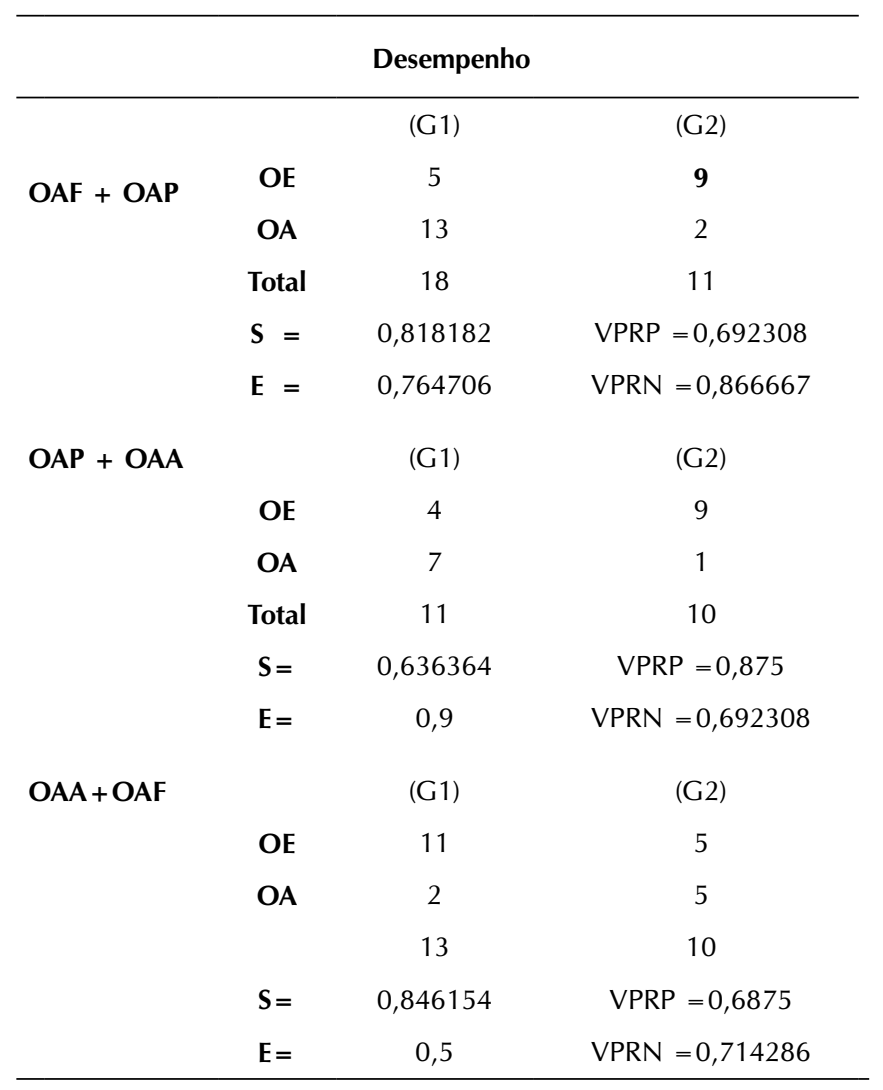

A organização dos dados para a determinação da sensibilidade e da especificidade das subescalas OAF e OAP possibilitou a delimitação do grupo de estudantes de alto risco para o desempenho insatisfatório na implementação dos cuidados de enfermagem para a prevenção das UP. As subescalas OAF e OAP tratam da orientação dos estudantes segundo o modo de agir diante do fracasso e no planejamento da ação, respectivamente.

\section{DISCUSSÃO}

Com base nos resultados verificou-se que o aumento da sensibilidade e especificidade deu-se com a combinação das escalas. No entanto, a escolha da melhor combinação levou em conta algumas considerações descritas a seguir.

É importante salientar que a subescala OAF se refere à orientação para a ação ou estado subsequente ao fracasso e a sua análise oportunizou a predição do déficit de desempenho nesta condição. Neste caso, significa dizer que os estudantes orientados para o estado apresentaram menor controle sobre as próprias reações frente ao fracasso recente, retratado no estudo pela avaliação insatisfatória na tarefa proposta. Cabe ressaltar que tal comportamento poderia interferir sobremaneira no planejamento e na execução das ações futuras, como a reavaliação dos fatores de risco ou prescrição de medidas preventivas, acarretando prejuízos para a continuidade da assistência.

Os resultados obtidos a partir da análise da subescala OAA possibilitaram uma aproximação maior com o envolvimento motivacional dos participantes do estudo na tarefa proposta. Os estudantes podem ser movidos por fatores motivacionais intrínsecos ou extrínsecos, como a auto-satisfação em adquirir conhecimento ou proporcionar benefícios para a clientela, a obtenção de notas, recompensas ou a aprovação dos pais e professores. Na orientação para a ação os estudantes têm objetivos claros e identificáveis quanto ao aprendizado motivado intrinsecamente e se tornam mais ativos na busca por estratégias de aprendizagem mais ajustadas às suas dificuldades. Por outro lado, na orientação para o estado evidencia-se a diminuição da motivação pela atividade no ambiente hospitalar, seja por falta de adaptação, pelo uso menor de estratégias de gestão do ambiente ou pela preferencia por cenários extra-hospitalares.

Tais dados ofereceram subsídios para identificar os estudantes mais vulneráveis aos interferentes ambientais do hospital, a partir da correlação feita entre a motivação intrínseca e o alcance dos objetivos da atividade em tela. O reconhecimento da relação entre motivação e intenções pessoais é um indicador importante para a manutenção da concentração no curso da ação, da persistência diante de situações adversas como o fracasso acadêmico e também para a conclusão da tarefa.

A subescala OAP investiga a orientação para a ação ou estado no planejamento e na decisão, ou seja, a coerência entre a intenção de fazer e a realização de fato. O julgamento clínico e pensamento crítico são elementos que permitem a analise da situação do cliente e oferecem subsídios para diagnóstico, tratamento e prognóstico. A pele íntegra exposta aos riscos, as causas, características e consequências da lesão quando instalada, o estado de saúde do cliente e a resposta terapêutica devem ser exaustivamente investigadas pelo 
estudante, acessando a capacidade crítico-reflexiva da situação e as bases de conhecimento. No que se refere à prevenção e ao tratamento das úlceras, a indecisão sobre a conduta ou a tomada de decisões precipitadas acarretam um plano de cuidados inadequado para o cliente. Ressalta-se que a elaboração imprecisa ou incompleta dos diagnósticos de enfermagem compromete a sistematização do cuidado de enfermagem e colabora para o agravamento da lesão.

Diante do exposto, foi definida a combinação mais pertinente para o evento em estudo. Houve destaque para os índices obtidos nas combinações entre as subescalas OAF/OAA $(S=0,84)$ e OAF/OAP $(S=0,81)$. Verificou-se no conjunto dos dados de OAF/OAP que a sensibilidade $(0,81)$ expressou as ocorrências positivas identificadas em ambos os grupos. A avaliação se complementou com a apresentação do valor preditivo negativo $(0,86)$, uma vez que. quanto mais sensível for o teste, maior será o valor preditivo negativo, ou seja, a quase garantia de que a condição investigada está ausente. Portanto, a aplicação das subescalas possibilitou o diagnóstico de estudantes em situação de risco para desempenho insatisfatório no estudo. Tal afirmativa é relevante para a identificação futura dos indicadores comportamentais entre os estudantes orientados para o estado no contexto da aprendizagem.

Nessa perspectiva, ressalta-se que o planejamento do cuidado de enfermagem implica em pensar e fazer. Tal dicotomia, potencializada por distrações intrínsecas ou extrínsecas, possibilita uma série de atitudes hesitantes ou permeadas pela preocupação, que interferem na ação concreta. Salienta-se que o êxito do cuidado de enfermagem na prevenção das úlceras por pressão pressupõe o uso de recursos que operam como guias de ação. O emprego da Sistematização da Assistência de Enfermagem (SAE) implica em etapas que se iniciam com o primeiro contato entre o enfermeiro e o cliente e se seguem até o alcance dos objetivos do mesmo ${ }^{(19)}$.

No caso dos estudantes analisados, a combinação entre OAF e OAP, oferece uma interpretação valiosa para o contexto assistencial, uma vez que tal conhecimento possa ser útil para compreensão do modo de agir em domínios específicos e as medidas preventivas são planejadas em coerência a priorização do atendimento individual às necessidades básicas do cliente. Nesse sentido, recomenda-se o uso desta combinação nas situações em que planejamento das ações tem maior peso.

Considerou-se que o modo de agir do estudante diante do fracasso acadêmico e o planejamento das ações expresso nos resultados das subescalas OAF e OAP são dimensões fundamentais para a aprendizagem do planejamento dos cuidados de enfermagem tanto em situações gerais quanto em situações específicas. O planejamento de uma tarefa de aprendizagem implica em pensar antecipadamente sobre as metas e os meios para alcançá-la. O controle da própria aprendizagem permite a detecção dos erros e desvios cometidos e essa reflexão conduz a avaliação e a modificação de um plano estratégico estabelecido. Refletir conscientemente sobre a aprendizagem ajudará a exercer maior controle sobre o modo de aprender e a compreender as lacunas dos processos e produtos $\operatorname{cognitivos}^{(20)}$.

A escolha da combinação entre OAF e OAP permitiu a recomendação do questionário HAKEMP 90 como recurso diagnóstico. A sua aplicação possibilitou a aproximação com elementos essenciais que devem ser enfaticamente abordados durante o processo de formação do enfermeiro, considerando o preparo para uma práxis qualitativa, integral, reflexiva e consciente.

Os dados apresentados evidenciaram que a atenção e a concentração interferiram no planejamento do cuidado de enfermagem, principalmente no que se refere à organização do pensamento, a priorização das ações e a tomada de decisão. De acordo com a análise, a sistematização do cuidado se constituiu como uma tarefa difícil para os estudantes orientados para o estado, fato corroborado pelos prejuízos na identificação dos fatores de risco, na elaboração dos diagnósticos de enfermagem, na prescrição e no registro dos cuidados preventivos para UP. Contudo, os estudantes orientados para a ação demonstraram-se mais ativos no processo de aprendizagem e apresentaram um plano de cuidados mais coerente com as etapas da sistematização da assistência.

Nessa perspectiva, para atender as demandas dos estudantes, foram utilizadas estratégias de intervenção norteadas para a identificação dos fatores que interferiram no desempenho. Para a abordagem individual das dificuldades e orientação adequada do grupo em tela, considerou-se a autoavaliação dos estudantes e o investimento na construção da aprendizagem ativa.

\section{CONCLUSÃO}

O delineamento do estudo contribuiu para a significação de conceitos seminais que alicerçam a aprendizagem do cuidado de enfermagem à luz dos pressupostos teóricos do controle da ação.

O diálogo com a enfermagem, em particular com a enfermagem fundamental, e o modo como a TCA se insere nessa área a coloca em uma condição adequada para promover a interseção entre a orientação ação-estado e o cuidado de enfermagem. O comportamento intencional que o estudante apresenta ao envolver-se com o cuidado prestado ao cliente hospitalar podem ser analisados sob a ótica do controle da ação. Essa discussão é imprescindível e abre mais um espaço para a reflexão sobre processos tipicamente humanos no campo do saber, da prática social de enfermagem e da condição de cuidar.

A compreensão dos elementos essenciais que caracterizam a aprendizagem possibilita a construção do processo de cuidar com competência e humanidade e, sobretudo mais qualitativo para o cliente. Cabe aqui enfatizar que o estudo contribuiu para a discussão sobre a relevância dos cuidados fundamentais de enfermagem prestados ao cliente no ambiente hospitalar, principalmente no momento da inserção e da adaptação do estudante em um cenário tão repleto de elementos capazes de causar distrações.

No âmbito do estudo, o uso da TCA oferece uma alternativa bastante significativa para a investigação das características individuais dos estudantes e dos vários fatores que interferem na implementação do cuidado. Neste contexto, percebe-se que prepará-los para o reconhecimento das condições que afetam a aprendizagem é essencial para a concretização das 
ações necessárias para o planejamento dos cuidados de enfermagem de modo mais coerente tanto para as ações mais simples quanto para as ações mais elaboradas.

Por outro lado, também possibilita que o professor conheça o papel das intenções e das distrações internas ou externas no desempenho do estudante. Assim sendo, o controle da ação torna-se uma ferramenta útil na medida em que a compreensão da orientação do estudante propicia subsídios para a elaboração de um plano de intervenções docentes fundamentado nos problemas apresentados por um grupo especifico.

\section{REFERÊNCIAS}

1. Kuhl J. A theory of self-regulation: action versus state orientation, self-discrimination, and some applications. Appl Psychol. 1992;41(2):97-129.

2. Kuhl J. A theory of action and state orientations. In: Kuhl J, Beckmann J. Volition and personality: Action versus state orientation. Germany: Gottingen; 1994. p. 9-46.

3. Blanes L, Duarte IS, Calil JA, Ferreira LM. Avaliação clínica e epidemiológica das úlceras por pressão em pacientes internados no Hospital São Camilo. Rev Assoc Med Bras. 2004;50(2):182-7.

4. Anselmi ML, Peduzzi M, França I Júnior. Incidência de úlcera por pressão e ações de enfermagem. Acta Paul Enferm. 2009;22(3):257-64.

5. Irion G. Feridas: novas abordagens, manejo clínico e atlas em cores. Rio de Janeiro: Guanabara Koogan; 2005.

6. Fernandes LM, Caliri MHL. Uso da escala de Braden e de Glasgow para identificação do risco para úlceras por pressão em pacientes internados em centro de terapia intensiva. Revista Latino-Am Enferm [periódico na internet]. 2008 [acesso em 20 nov 2010]:16(6):973-978. Disponível em: http://www. scielo.br/pdf/rlae/v16n6/pt_06

7. Kuhl J, Kazén-Saad M. Volition and self-regulation: memory mechanisms mediating the maintenance of intentions, In Hershberger WA. Volitional action: conation and control. North Holland: Elsevier Science Publishers; 1989. p. 387-407.

8. Papantoniou G, Moraitou D, Katsadima E, Dinou M. Control de la acción y disposición a la esperanza: um studio de su incidencia en la autorregulación del aprendizaje. Electron J Research Educational Psychology. 2010;8(1):5-32.

9. Coll C, Palacios J, Marchesi A. Desenvolvimento psicológico e educação: psicologia da educação. Porto Alegre: Artes Médicas; 1996.

10. Kuhl J. Volitional mediators of cognition- behavior consistency: self-regulatory process and action versus state orientation. In: Kuhl J, Beckmann J. Action control: from cognition to behavior. Heidelberg: Springer -Verlag; 1985. p. 89 - 100.

11. Kuhl J. Action vs state orientation as a mediator between motivation and action. In: Hacker W, Volpert W, Von Cranach M. Cognitive and motivacional aspects of action. Amsterdan: North Holland; 1982. p. 67-85.

12. Kuhl J. Action and state orientation: Psychometric properties of the action control scales (ACS-90). In: Kuhl J, Beckmann J. Volition and personality: action versus state orientation. Gottingen: Hogrefe; 1994. p. 47-59.

13. Brislin RW. Back translation for cross cultural research. J Cross Cult Psychol. 1970;1(3):185-216.

14. Castelo Branco EMS. O controle das ações do estudante de enfermagem na implementação dos cuidados preventivos para úlceras por pressão. Rio de Janeiro. Tese [Doutorado em enfermagem] - Escola de Enfermagem Anna Nery; 2007.

15. Medronho RA, Carvalho DM, Bloch KV, Luiz RR, Werneck GL. Epidemiologia. São Paulo: Atheneu; 2005.

16. Fletcher RH, Fletcher SW, Wagner EH. Epidemiologia clínica: elementos essenciais. 4. ed. Porto Alegre: Artes Médicas; 2006.

17. Nassar SM. Tratamento de Incerteza: Sistemas Especialistas Probabilísticos. 2007 [acesso em 20 jul 2008]. Disponível em: http://www.inf.ufsc.br/silvia/disciplinas/sep/material_didatico.pdf

18. Kuhl J. Self-regulatory processes and action versus state orientation. In: Kuhl J, Beckmann J. Action Control: From Cognition to Behavior. Berlin: Springer-Verlag; 1985. p 119-120.

19. Leopardi MT. Teoria e método em assistência de enfermagem. 2. ed. Florianópolis: Ed. Soldasoft; 2006. 306p.

20. Pozo JI. Aprendizes e mestres: a nova cultura da aprendizagem. Porto Alegre: Artmed; 2002. 